\title{
EDITORIAL
}

\section{El año que vivimos en peligro: Un antes y después para la Educación en Negocios}

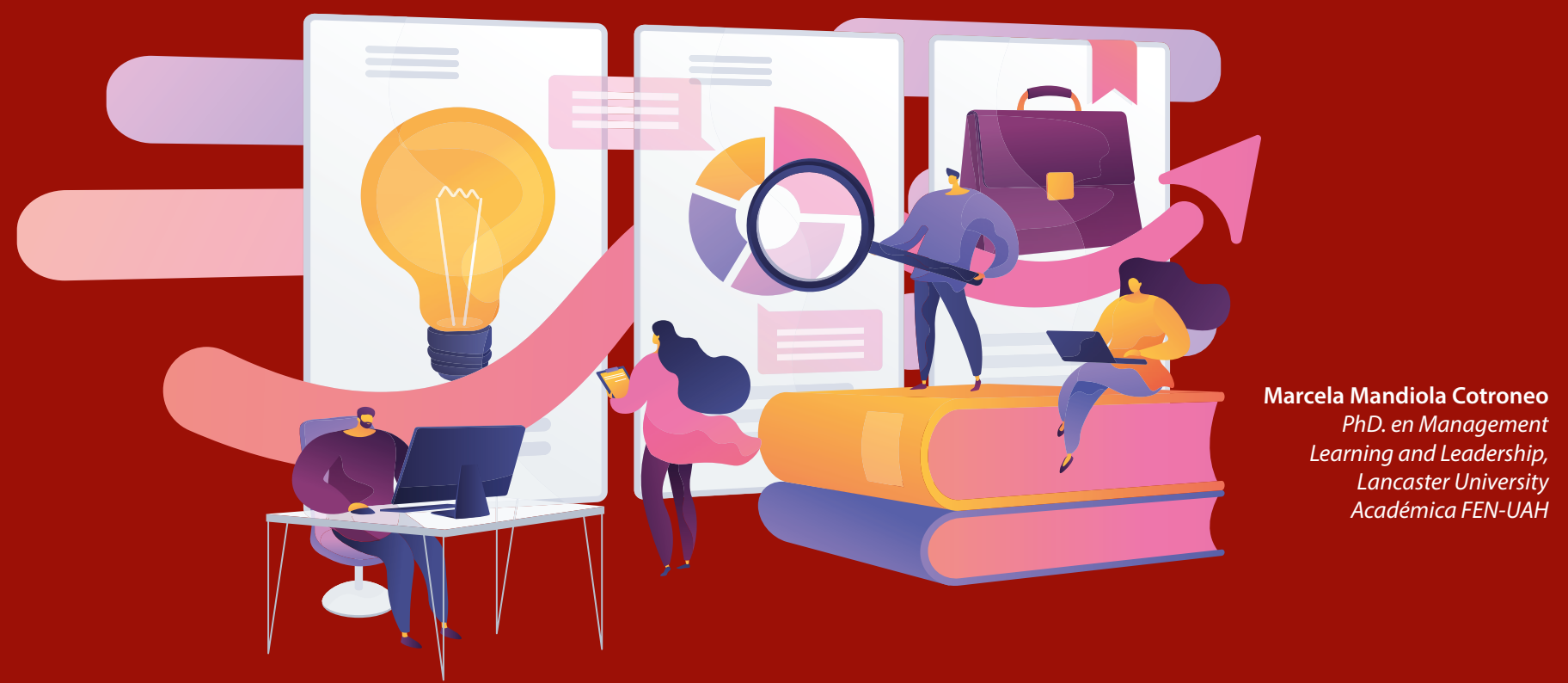

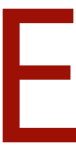
El año 2020 ha venido a establecer un quiebre de proporciones a la cotidianeidad de la vida. Nuestro modo de habitar, de producir, de relacionarnos, se ha visto interrumpido, más bien detenido, y con ello nos hemos enfrentado a la abrupta y obligatoria oportunidad de observarnos y reflexionar. Lo que vivimos hoy no solo se trata de una emergencia sanitaria o un problema de salud pública. En un mundo globalizado que se ha articulado a través de relaciones de mercado dinámicas y competitivas, la detención y el confinamiento han causado estragos. Lo que emerge como más grave aún, es la falta de alternativas para organizar los modos de sostener la materialidad de la vida a consecuencia directa de una concepción económica monocorde y hegemónica que se quedó sin 'propuesta de valor' ante una 'externalidad' que nunca modeló. Vis a vis, las estrategias de gestión surgidas al alero de dicha visión económica, y que han sido amplia y hegemónicamente difundidas a través de la educación en negocios tradicional, alcanzan también su límite. Basta una mirada superficial a los contenidos y metodologías de los programas académicos asociados a la disciplina de la administración para darnos cuenta que seguimos educando para una mundo que ya no existe. Urge, apremia, más que nunca, revisar, revisitar y desafiar lo que hemos entendido hasta ahora como educación en negocios.

Los cuestionamientos a la educación en negocios se discuten hace tiempo en el Norte Global. Las revisiones van desde el lamento por la pérdida de los 'objetivos superiores' en un par de 'manos atadas' de Rakesh Khurana (2007); pasando por intentos de instalar una enseñanza 'responsable' de la gestión (www.unprme.org) impulsada por las agencias internacionales; el develamiento de la 'fabricación de la moral' en la enseñanza de los negocios que hizo Michel Anteby (2013); hasta la provocativa propuesta de Martin Parker (2018) y su llamado a derribar las escuelas de negocios. Sin embargo, poco y nada de eso toca a las impermeables instalaciones latinoamericanas que se han caracterizado más por reproducir una práctica y una didáctica importada que por desarrollar o proponer desde su propia mirada (IbarraColado, 2006; Muñoz, 2011; Mandiola, 2013). Aquí queremos enfatizar no sólo la búsqueda de propuestas de contenido propio para la administración, la gestión o los estudios organizacionales pensados desde nuestra geopolítica, si no también a la problematización de la educación formal en dichas prácticas disciplinares. En otras palabras, no solo el qué, de modo especial la pregunta es por el cómo y el para qué.

Una búsqueda simple entre las publicaciones científicas que se organizan bajo los descriptores de educación en negocios o educación en management arroja pingües contribuciones desde nuestro subcontinente en comparación con la abultadísima producción, agrupada en 


\section{"Para la educación en negocios no queda más que revisitarse o desaparecer. Con este número especial esperamos contribuir a esta problematización"}

revistas temáticas y libros especializados que ostenta el mundo anglosajón y europeo. En términos de dar cuenta de una mirada comprensiva de lo que se ha escrito en Latam se hizo necesario realizar una búsqueda que comprendió los últimos 20 años de modo de contar con evidencia que permitiera referirse a ciertas tendencias. En este sentido, es posible identificar tres grandes grupos de publicaciones, a saber, aquellas que se enmarcan dentro de la corriente principal y desde allí buscan estilizar la práctica de las escuelas de negocios tal y como se realiza en el resto del orbe (Reficco et al; 2019; Alvarado, et al, 2018; Henley et al; 2017; Araya-Castillo y Escobar-Farfán, 2015; Koljatic y Silva, 2015; Orozco y Villaveces, 2015); por otro lado, las publicaciones que evidencian investigación con miradas alternativas que problematizan la actual práctica de educación en negocios (Mandiola, 2019; Gutierrez, en prensa; Gantman y Parker, 2006; Alcadipani y Caldas, 2012; Muñoz, 2011) y por último, aquellos que apuntan a realizar una revisión histórica y contextual de la educación en negocios en Latinoamérica (Wanderley y Barros, 2019; Mandiola y Varas, 2018; Cook y Alcadipani, 2015; Alca- dipani y Bertero, 2012). Con todo, se trata de un ejercicio reflexivo y autocrítico que ha ocupado hasta ahora muy pocos de los esfuerzos de la academia latinoamericana en administración y negocios, con una importante concentración en las investigaciones realizadas en Brasil y Colombia.

Considerando este escenario nuestra publicación Gestión y Tendencias de la Facultad de Economía y Negocios de la Universidad Alberto Hurtado, ha decidido dedicar un número especial a este importante tema de la Educación en Negocios. Lo nuestro es una primera invitación a instalar esta discusión en nuestro país, en conversación también con Latinoamérica. Nuestro llamado fue gentilmente respondido por un grupo de académicos y académicas desde México, Colombia, Inglaterra y por supuesto nuestro país. Este número especial aglutina las reflexiones contingentes de Eduardo Acuña (U Chile) por un lado, y Alejandro Saldaña (U, Veracruzana, México) por el otro, quienes problematizan la situación actual de la educación en negocios en contextos de pandemia hurgando en sus aciertos y desaciertos y en los desafíos que se han abierto para la práctica. Por su parte, las profesoras Mary Vera, Zuray Melgarejo y Claudia Niño (U. Nacional de Colombia) nos trasladan a una experiencia concreta de docencia en pandemia en la especialidad de contabilidad, reflexionando sobre el impacto de eventos críticos inesperados sobre las experiencias pedagógicas en nuestra disciplina. Alvaro Espejo (IESE U. Andes) se pregunta directamente si es el momento de cambiar lo que conocemos como educación en negocios o más bien reformular sus predicamentos y prácticas en pos de una adaptación a las demandas actuales. Finalmente, Juan Pablo Subiabre (U. de Bristol, UK) nos adentra en la ya discutida tensión entre relevancia y rigor cuando se habla de educación en negocios y en las estrecheces de dicho debate frente a situaciones contextuales que exigen una mirada completamente distinta a la práctica.

Sin más, la educación en negocios tal como la hemos conocido y llevado adelante hasta hoy, se enfrenta a una crisis de proporciones. No sólo es una más de las experiencias afectadas por la crisis sanitaria, social y económica que nos aqueja, se trata además de uno de los engranajes fundamentales del mundo que hoy está en retirada. Para la educación en negocios no queda más que revisitarse o desaparecer. Con este número especial esperamos contribuir a esta problematización.

\section{Referencias}

Alcadipani, R. y Bertero, C. (2012) The Cold War and the Teaching of Management in Brazil: the case of FGV-EAESP. Rae-Revista de Administracao de Empresas 52 (3): 284-99.

Alcadipani, R. y Caldas, M. (2012),"Americanizing Brazilian management", Critical perspectives on international business, Vol. 8 Iss 1 pp. 37 - 55

Alvarado, G.; Howard, T.; Lynne, T. y Wilson, A. (2018) Latin America: Management education's growth and future pathways. Global Focus: The EFMD Business Magazine Anteby, M. (2013) Manufacturing Morals. The values of silence in Business School Education. The University of Chicago Press. Chicago.

Araya-Castillo, M. y Escobar-Farfán, M. (2015) Personalidad de marca de las escuelas de negocios en Chile: Propuesta de modelo. AD-minister No. 27 julio-diciembre pp. $53-73$

Cook, B. y Alcadipani, R. (2015) Toward a Global History of Management Education: The Case of the Ford Foundation and the Sao Paulo School of Business Administration Brazil. Academy of Management Learning \& Education 14 (4): 482-99

Gantman, E. y Parker, M. (2016) Comprador management? Organizing management knowledge in Argentina (1975-2003). Critical Perspectives on International Business Gutierrez, F. (en prensa) (Re)produciendo una realidad sin sindicatos. Las Escuelas de Negocio en Chile. Latin American Perspectives

Henley, A.; Contreras, F.; Espinosa, J.C. y Barbosa, D. (2017) Entrepreneurial Intentions of Colombian Business Students Planned Behaviour, Leadership Skills and Social Capital. International Journal of Entrepreneurial Behaviour \& Research 23 (6, SI): 1017-32.

Ibarra-Colado, E. (2006) "Organization Studies and Epistemic Coloniality in Latin America: Thinking Otherness from the Margins." Organization 13(4): 463 - 488.

Koljatic, M. y Silva, M. (2015) Do Business Schools Influence Students' Awareness of Social Issues? Evidence from Two of Chile's Leading MBA Programs. Journal of Business Ehics 131 (3): 595-604

Khurana, R. (2007) “From Higher Aims to Hired Hands: The Social Transformation of American Business Schools and the Unfulfilled Promise of Management as a Profession". Princeton University Press.

Mandiola Cotroneo, M. (2013) Management education in Chile: From politics of pragmatism to (im)possibilities of resistance. Universitas Psychologica, 12(4), 1085-1098. Mandiola, M. (2019) "Problematizando la Educación en Negocios en Chile: una práctica (no) fisurada." Revista Stultifera, Vol 2 num 2 pp. $59-73$

Mandiola, M. y Varas, A. (2018) “Educar es gobernar" Explorando los inicios del managerialismo masculino En la academia chilena. Revista de Ciencias Sociales, DS-FCS vol. 31, n.o 43, julio-diciembre 2018, pp. 57-78.

Muñoz, R. (2011) Formar en administración. Por una nueva fundamentación filosófica. Fondo Editorial Universidad EAFIT. Medellín

Orozco, L.A. y Villaveces, J.L. (2015) Heterogeneous Research Networks in Latin American Schools of Business Management. Academia-Revista Latinoamericana de Administracion 28 (1): 115-34.

Parker, M. (2018) Shutdown Business Schools. London. Pluto Press.

Reficco, E.; Jaen, M.H. y Trujillo, C. (2019) Beyond Knowledge: A Study of Latin American Business Schools' Efforts to Deliver a Value-Based Education. Journal of Business Ethics

Wanderley, S. y Barros, A. (2019) The Alliance for Progress, modernization theory, and the history of management education: The case of CEPAL in Brazil. Management Learning 1-8 